\title{
Hepatitis B-related Acute-on-Chronic Liver Failure Induced by Hepatotropic Viral insult is Associated with Worse Prognosis Than that Induced by Non- Virus Insult
}

Jing Liang ( $\sim$ haolele77@sina.com )

Tianjin Third Central Hospital https://orcid.org/0000-0001-5114-9030

Lei Liu

Tianjin Third Central Hospital

Yingying Cao

Tianjin Third Central Hospital

Qian Zhang

Tianjin Third Central Hospital

Fang Liu

Tianjin Third Central Hospital

Chen $\mathrm{Yu}$

Capital Medical University Youan Hospital

Hua Liu

Tianjin Third Central Hospital

Zhongping Duan

Capital Medical University Youan Hospital

Manman Xu

Capital Medical University Youan Hospital

Shaojie Xin

5th Medical Center of Chinese PLA General Hospital

Shaoli You

5th Medical Center of Chinese PLA General Hospital

Fangjiao Song

5th Medical Center of Chinese PLA General Hospital

Jun Li

Tianjin Third Central Hospital

Tao Han

Tianjin Third Central Hospital 
Research article

Keywords: Acute-on-chronic liver failure, Hepatitis B virus reactivation, Precipitating events,Prognosis

Posted Date: February 1st, 2021

DOl: https://doi.org/10.21203/rs.3.rs-191136/v1

License: (c) (i) This work is licensed under a Creative Commons Attribution 4.0 International License. Read Full License

Version of Record: A version of this preprint was published at BMC Infectious Diseases on December 1st, 2021. See the published version at https://doi.org/10.1186/s12879-021-06974-z. 


\section{Abstract}

Objective: The manifestations and prognoses of acute-on-chronic liver failure (ACLF) with different precipitating events remain heterogeneous. We aimed to investigate the characteristics and prognosis of patients with hepatotropic viral insult (HVI)-induced hepatitis B-related ACLF (HBV-ACLF).

Methods: 452 patients with confirmed diagnosis of ACLF were screened in three medical centers in China, and 203 HBV-ACLF patients with definite acute precipitating events were retrospectively analyzed.

According to the precipitating events, HBV-ACLF patients induced by HBV reactivation and super-infection with HAV were classified as the hepatotropic viral insult group and those induced by other factors, as the non-virus insult ( $\mathrm{NVI}$ ) group. The clinical characteristics, predictive scoring model, and prognosis of the two groups were compared.

Results: Hepatitis B virus reactivation accounted for the largest proportion (39.9\%) among all precipitating events. Exacerbation time frame of the HVI group was significantly longer than that of the $\mathrm{NVI}$ group (20 days vs. 10 days, $\mathrm{P}<0.001$ ). Comparison of intergroup prognosis showed that there was no significant difference in the 28 -day mortality $(20.9 \%$ vs. $13.7 \%, P=0.125)$, while the 90 -day and 1 -year mortality in the HVI group were higher than those in the NVI group ( $36.3 \%$ vs. $24.4 \%, \mathrm{P}=0.014 ; 39.5 \%$ vs. $27.5 \%, P=0.020$, respectively). In the HVI group, the lactic acid-free APASL-ACLF Research Consortium(AARC) had the highest predictive value for 90-day mortality (0.741).

Conclusions: The 90-day and 1-year survival rate was lower in HBV-ACLF patients induced by HVI than by $\mathrm{NVI}$. The lactate-free AARC score was a better predictor of short- and long-term prognosis in patients with HVI-induced HBV-ACLF.

Trial registration: ChiCTR, ChiCTR1900021539 . Registered 26 February 2019 Retrospectively registered, http://www.chictr.org.cn/showprojen.aspx?proj=36342

\section{Introduction}

Acute-on-chronic liver failure (ACLF) is a complex clinical syndrome with acute deterioration of liver function, organ failure, and high short-term mortality, which is considered distinct from acute decompensation of cirrhosis[1, 2]. At present, because of the differences in the etiology of liver disease in Europe, USA, and the Asia Pacific region, there is no consensus yet on the diagnostic criteria of ACLF[3]. Given that patients with ACLF represent a heterogeneous population, previous studies focused on the clinical characteristics of ACLF patients with different precipitating events. However, the conclusions were inconsistent. Shi et al. reported that the 90-day and 1-year survival time of ACLF caused by extrahepatic insults were lower than that caused by hepatic injury[4]. However, Yin et al. suggested that there was no difference in short-term mortality of patients with ACLF between hepatic and extrahepatic precipitating events[5]. Fernandez et al. suggested that the risk of aggravation in patients with bacterial infectioninduced ACLF was significantly higher than that of patients without bacterial infection, and the 90-day accumulative survival of the former was significantly lower than the latter[6]. It should be noted that all 
the above studies were aimed at ACLF populations based on different criteria, wherein the etiology of ACLF is not specifically defined. In China, chronic hepatitis B is the main cause of ACLF[7]. Hepatotropic viral infection includes hepatitis B virus (HBV)reactivation and super-infection with hepatitis A virus (HAV) or hepatitis E virus (HEV) and is the major cause of acute insults for precipitating ACLF[8]. Studies on the characteristics and prognosis of the HBV-ACLF patients induced by hepatotropic viral infection are limited. Therefore, we aimed to analyze the characteristics and prognosis of HBV-ACLF patients with definite hepatotropic viral infection from multiple centers in China, according to the enrolment standards of the Asian Pacific Association for the Study of Liver (APASL) consortium.

\section{Patients And Methods Study population}

Patients who fulfilled the APASL ACLF criteria from a retrospective cohort referred between December 2012 and February 2019 in The Third Central Hospital of Tianjin, Beijing You'an Hospital Affiliated to Capital Medical University, and The Fifth Medical Center of Chinese PLA General Hospital were screened. According to APASL recommendations[8], 452 patients who were diagnosed with ACLF failure, defined as acute severe hepatic damage (total bilirubin $[\mathrm{TBIL}] \geq 5 \mathrm{mg} / \mathrm{dL}$ and international normalized ratio [INR] $\geq$ 1.5 or prothrombin activity $[\mathrm{PTA}] \leq 40 \%$ ) accompanied by ascites and/or hepatic encephalopathy [HE] within 4 weeks, were screened. The exclusion criteria were as follows: 1) age < 18 years; 2) non HBVrelated liver disease; 3 ) combined with hepatocellular carcinoma (HCC) or extrahepatic malignancies; 4) combined with HCV or HIV infection; 5) patients with no definite precipitating event; 6) those who had received a liver transplant; and 7) those whose hospital stay was $<1$ day.

The diagnostic criteria of chronic hepatitis B refers to the Guideline of Prevention and Treatment for Chronic Hepatitis B of China[9]: positive for the HBV surface antigen $\geq 6$ months. Cirrhosis was diagnosed based on previous liver biopsy results, clinical evidence of previous laboratory tests, and endoscopic (esophageal and gastric varices) and radiological imaging of portal hypertension. Overt ascites was diagnosed by ultrasonography. Hepatic encephalopathy was defined and graded based on the West Haven criteria[10]. Exacerbation time refers to the time from the precipitating event to the occurrence of liver failure.

\section{Data Collection}

We collected the following clinical and demographic information in a retrospective database datasheet: age, sex, history, exacerbation time, precipitating events, laboratory parameters, and mortality at 28 days, 90 days, and 1 year. For all patients, laboratory data were collected at days 1, 7, 14, and 28 after diagnosis of ACLF.

\section{Definition Of Precipitating Events And Grouping}


The enrolled patients had a record of definite precipitating events (PEs). The eight potential PEs were defined as follows:

1) HBV reactivation is defined as an increase in HBV DNA level by $\geq 2 \mathrm{lg} \mathrm{IU} / \mathrm{mL}$ from baseline or reappearance of HBV DNA viremia or HBsAg in those with previously detectable HBV DNA or HBsAg owing to withdrawal of antiviral drugs and resistance to nucleotide analogues or after treatment of immunosuppressive and chemotherapeutic drugs[9, 11];

2) Super-infection with HAV and HEV was diagnosed by the presence anti-HAV/HEV serum immunoglobulin IgM and IgG[12];

3) Hepatotoxic drugs' insult was defined as the use of hepatotoxic drugs such as antituberculotic drugs, non-steroidal anti-inflammatory drugs, or herbal medicine within 3 months before diagnosis of ACLF, and a strong temporal relationship between exposure to the drug and development of ACLF[13];

4) Active alcohol consumption was defined as having more than 14 drinks per week in women and more than 21 drinks per week in men, within 6 months prior to admission[2];

5) Upper gastrointestinal bleeding (UGIB)was defined as active bleeding due to esophageal and gastric varices within 1 month before diagnosis of ACLF;

6) Bacterial infection was defined as previously described[14] including spontaneous peritonitis, intestinal infections, lung infections, urinary tract infections, or bacteremia, when they were detected prior or at the time of diagnosis of ACLF;

7) Overwork was defined as a patient's self-reported history of moderate intensity physical and mental work beyond the normal working hours within 4 weeks before the diagnosis of $\operatorname{ACLF}[15,16]$.

8) Surgery was defined as the surgical operation under general anesthesia.

Acute precipitating events were categorized into two groups. HBV reactivation and super-infection with $\mathrm{HAV}$ or HEV were classified as the hepatotropic viral insult (HVI) group and the other factors as the control group or non-virus insult (NVI) group.

\section{Calculation of liver scoring systems}

The following five prognostic models were used to analyze the prognosis of ACLF patients with different PEs included: Child-Pugh score (CTP: range, 0-15)[17]; Model for End-Stage Liver Disease (MELD: range, 6-40)[18]; Chronic Liver Failure-Sequential Organ Failure Assessment (CLIF-SOFA); CLIF-ConsortiumACLF (CLIF-C-ALCF); and APASL-ACLF Research Consortium (AARC). CLIF-SOFA score (range, 0-24) consists of six organ systems and is proposed to evaluate organ failures in ACLF patients[2]. CLIF-CACLF score is adjusted based on the CLIF-SOFA scale and calculated as follows: $10 \times[0.33 \times$ CLIF-OFs + $0.04 \times$ Age $+0.63 \times \operatorname{Ln}($ WBC count $)-2][19]$. AARC-ACLF score is recommended by APASL as a reliable 
prediction model for ACLF patients, which is measured by HE, serum bilirubin, INR, lactate, and creatinine[20]. However, in this study, we used lactate-free AARC instead because the serum lactate was not a routine test parameter in some areas.

\section{Statistical Analysis}

Continuous variables were expressed as mean \pm standard deviation (SD) or median with interquartile range (IQR). Continuous data between two groups were compared by Student's $t$-test or Mann-Whitney $U$ test. Comparison of categorical variables was carried out by chi-square test. The 28-day, 90-day, and 1year mortality curves of different groups were analyzed using the log-rank test. Predictors for the 28-day and 90-day mortality were analyzed by univariate and multivariate Cox regression model. Dynamic changes of continuous variables between groups were compared using repeated measurements. ROC curve was used to explore the prediction model. Statistical analyses were performed using SPSS (23.0; SPSS, Inc., Chicago, IL). P < 0.05 was considered to indicate statistical significance.

\section{Results}

\section{Clinical Characteristics}

Of 452 patients with ACLF, 203 patients with HBV-ACLF with definite PEs were finally included (Fig. 1). Among the included patients, 186 were identified at admission, and 17 patients with cirrhosis developed ACLF within 14 days after admission. The mean age of the patients was 47.7 years. Jaundice and fatigue were the main symptoms of all patients. The mean time of disease deterioration was 14 days. The 28-day, 90-day, and 1-year mortality of all ACLF patients were $18.2 \%, 32 \%$, and $35.5 \%$, respectively. Types of PEs among all patients are listed in Table 1. HBV reactivation accounted for the largest proportion (39.9\%) in all precipitating events, which included the recurrence of HBV by withdrawal of antiviral drugs (28.1\%) and application of immunosuppressive drugs, chemotherapeutic drugs (5.9\%), or viral mutations (5.9\%).Among the NVI group, the proportion of acute exacerbation caused by bacterial infection was the highest (29.6\%), followed by drug insult (10.3\%), active alcohol drinking (10.3\%), and overwork (7.9\%). In addition, one patient(0.5\%) underwent surgical operation. 
Table 1

Etiology of precipitating events

\begin{tabular}{|ll|}
\hline Precipitating events & Frequency \\
\hline Hepatotropic viral insult(N = 82) & \\
\hline HBV reactivation, no (\%) & $81(39.9)$ \\
\hline Super-infection with HAV, no (\%) & $1(0.5)$ \\
\hline Non-virus insult(N = 121) & \\
\hline Bacterial infection, no (\%) & $60(29.6)$ \\
\hline Drug insult.no (\%) & $21(10.3)$ \\
\hline Active alcohol drinking, no (\%) & $21(10.3)$ \\
\hline Overwork, no (\%) & $16(7.9)$ \\
\hline Upper gastrointestinal bleeding, no(\%) & $2(1.0)$ \\
\hline Surgery, no(\%) & $1(0.5)$ \\
\hline
\end{tabular}

\section{Comparison of clinical characteristics between the HVI and NVI groups}

Comparison of the clinical characteristics between the HVI and NVI group are shown in Table 2. There were no significant intergroup differences in age, sex, and history of cirrhosis. Exacerbation time frame in the HVI group was significantly longer than that in the NVI group (20 days vs. 10 days, $P<0.001)$. At the time of ACLF diagnosis, the level of albumin in the HVI group was significantly higher than that in the NVI group, and there was no significant difference in other parameters of hepatic and renal function. There were no significant intergroup differences in the incidence of organ failure, baseline Child-Pugh, MELD, CLIF-SOFA, CLIF-ACLF, and lactate-free AARC scores. 
Table 2

Comparison of clinical features and prognosis between hepatotropic viral insult and non-virus insult groups

\begin{tabular}{|c|c|c|c|c|}
\hline & Total $(n=203)$ & $\mathrm{HVI}(\mathrm{n}=82)$ & $\operatorname{NVI}(n=121)$ & P Value \\
\hline Age,years & $47.7 \pm 11.4$ & $49.3 \pm 9.6$ & $46.6 \pm 12.4$ & 0.087 \\
\hline Male,no(\%) & $161(79.3)$ & $64(73.1)$ & $97(80.2)$ & 0.715 \\
\hline Cirrhosis,no(\%) & $134(66.0)$ & $54(65.8)$ & $80(66.1)$ & 0.969 \\
\hline Exacerbation time frame,days & $14(23)$ & $20(20)$ & $10(14)$ & 0.003 \\
\hline \multicolumn{5}{|l|}{ Laboratory data } \\
\hline ALT, IU/L & $185.0(470.5)$ & $151.7(535.7)$ & $219(472.5)$ & 0.927 \\
\hline AST, IU/L & 203.3(374.4) & $184.5(460.4)$ & $207.0(394.1)$ & 0.785 \\
\hline Albumin,g/l & $29.9 \pm 5.1$ & $31.5 \pm 4.8$ & $28.9 \pm 5.1$ & 0.000 \\
\hline GGT, IU/L & $85.0(83.8)$ & $92.0(101)$ & $82.0(77.5)$ & 0.091 \\
\hline TB,umol/L & 263.3(224.4) & 285.8(215.4) & $252.4(216.6)$ & 0.216 \\
\hline $\mathrm{Na}, \mathrm{mmol} / \mathrm{L}$ & $135.1(5.5)$ & $134.6(5.5)$ & $135.2(5.8)$ & 0.788 \\
\hline Creatinine,umol/L & $72.0(37.0)$ & $68(36.5)$ & $76(38.5)$ & 0.195 \\
\hline WBC count, $10^{9} / \mathrm{L}$ & $6.2(4.5)$ & $5.8(8.6)$ & $6.8(4.2)$ & 0.227 \\
\hline NEUT, \% & $72.0(14.6)$ & 71.4(15.3) & $72.0(15.0)$ & 0.880 \\
\hline Platelet,count, $10^{9} / \mathrm{L}$ & 79(70) & 79(117) & $80(61)$ & 0.845 \\
\hline PTA,\% & $33.8(13.6)$ & 33.6(14.3) & $34(13.2)$ & 0.896 \\
\hline INR & $2.1(0.8)$ & $2.1(0.9)$ & $2.1(0.8)$ & 0.707 \\
\hline \multicolumn{5}{|l|}{ Severity score } \\
\hline CTP & $11.0(2)$ & $11.0(2)$ & $11.0(2)$ & 0.134 \\
\hline MELD & $23.6 \pm 5.4$ & $23.7 \pm 6.1$ & $23.5 \pm 4.9$ & 0.830 \\
\hline CLIF-SOFA & $7(1)$ & $7(2)$ & $7(1)$ & 0.660 \\
\hline lactate free AARC & $7(1)$ & $9(2)$ & $8(3)$ & 0.738 \\
\hline CLIF-ACLF & $39.2(12.7)$ & $41.3(11.7)$ & $38.3(12.8)$ & 0.188 \\
\hline \multicolumn{5}{|l|}{ Cumulative mortality } \\
\hline 28-day, no(\%) & $37(18.2)$ & 19(20.9) & 18(13.7) & 0.125 \\
\hline 90-day, no(\%) & $65(32.0)$ & $33(36.3)$ & $32(24.4)$ & 0.020 \\
\hline
\end{tabular}




\begin{tabular}{|lllll|}
\hline & Total $(\mathrm{n}=203)$ & $\mathrm{HVI}(\mathrm{n}=\mathbf{8 2})$ & $\mathrm{NVI}(\mathrm{n}=121)$ & P Value \\
\hline 1-year, no(\%) & $72(35.5)$ & $36(39.5)$ & $36(27.5)$ & 0.014 \\
\hline
\end{tabular}

3. Comparison of prognosis and mortality between hepatotropic viral insult group and non-virus insult group

There was no difference in the 28 -day mortality $(20.9 \%$ vs. $13.7 \%, P=0.125)$ between the two groups. However, the 90-day and 1-year mortality were significantly higher in the HVI group than the NVI group (36.3\% vs. $24.4 \%, P=0.020 ; 39.5 \%$ vs. $27.5 \%, P=0.014$; respectively) (Table 2, Fig. 2 ). The 90 -day and 1 year survival of patients with HVI-induced ACLF were lower than those induced by other insults (Fig. 3).

Predictors of 28-day and 90-day mortality in the two groups were analyzed by multivariate cox regression. Cirrhosis, bilirubin, and PTA were independent predictors of 28-day mortality, while age, PTA, and creatinine (Cr) were independent predictors for 90-day mortality in the $\mathrm{HVI}$ group. Only creatinine was an independent predictor of 90-day mortality in the NVI group (Table 3). 
Table 3

Risk factors associated with 28-day and 90-day mortality by a Multivariate COX's Hazard Model in HVI and NVI groups

\begin{tabular}{|c|c|c|c|c|c|c|}
\hline \multicolumn{3}{|c|}{ 28-day mortality } & \multicolumn{4}{|c|}{ 90-day mortality } \\
\hline Variables & Hazard Ratio & $95 \% \mathrm{Cl}$ & P Value & Hazard Ratio & $95 \% \mathrm{Cl}$ & P Value \\
\hline \multicolumn{7}{|c|}{$\mathrm{HVI}(\mathrm{n}=82)$} \\
\hline Cirrhosis & 2.179 & $0.061-0.870$ & 0.030 & 0.104 & $0.355-2.101$ & 0.747 \\
\hline Age & 4.694 & $0.989-1.122$ & 0.107 & 8.559 & $1.024-1.125$ & 0.003 \\
\hline TBil & 2.595 & $1.001-1.007$ & 0.020 & 2.305 & $0.999-1.004$ & 0.129 \\
\hline PTA & 5.373 & $0.878-0.993$ & 0.029 & 6.145 & $0.900-0.988$ & 0.013 \\
\hline $\mathrm{Cr}$ & 4.759 & $0.998-1.007$ & 0.349 & 8.622 & $1.002-1.008$ & 0.003 \\
\hline $\mathrm{Na}$ & 0.877 & $0.951-1.157$ & 0.335 & 0.554 & $0.920-1.138$ & 0.457 \\
\hline WBC & 0.929 & $0.887-1.173$ & 0.781 & 0.000 & $0.889-1.125$ & 0.999 \\
\hline Neut & 0.077 & $0.951-1.059$ & 0.903 & 0.462 & $0.948-1.126$ & 0.497 \\
\hline \multicolumn{7}{|c|}{$\mathrm{NVI}(n=121)$} \\
\hline Age & 0.498 & $0.974-1.058$ & 0.480 & 0.012 & $0.967-1.031$ & 0.912 \\
\hline PTA & 0.004 & $0.908-1.109$ & 0.948 & 0.078 & $0.919-1.065$ & 0.780 \\
\hline $\mathrm{Cr}$ & 0.760 & $0.992-1.021$ & 0.383 & 4.330 & $1.001-1.024$ & 0.037 \\
\hline $\mathrm{Na}$ & 0.109 & $0.931-1.105$ & 0.741 & 0.011 & $0.940-1.071$ & 0.918 \\
\hline WBC & 0.104 & $0.940-1.089$ & 0.748 & 0.025 & $0.940-1.054$ & 0.873 \\
\hline Neut & 1.389 & $0.979-1.089$ & 0.239 & 2.832 & $0.995-1.075$ & 0.092 \\
\hline INR & 1.358 & $0.601-7.422$ & 0.244 & 0.513 & $0.526-3.980$ & 0.474 \\
\hline
\end{tabular}

The clinical parameters of the 1st, 7th, and 14th day after ACLF diagnosis were measured dynamically and compared between patients who died or survived at 90 days in both groups. TBil, INR, white blood cell count (WBC), and serum sodium ( $\mathrm{Na}$ ) and creatinine levels showed significant dynamic changes between the deceased and survived patients in the NVI group within 2 weeks after admission. However, these parameters were not significantly different in the HVI group (Table 4 and Fig. 4). 
Table 4

Dynamic changes of parameters within 14 days after ACLF diagnosis in in $\mathrm{HVI}$ and NVI groups

\begin{tabular}{|lll|}
\hline Variables & F & P value \\
\hline HVI $(n=82)$ & & \\
\hline TB & 2.98 & 0.090 \\
\hline PTA & 5.876 & 0.019 \\
\hline INR & 2.763 & 0.102 \\
\hline Cr & 2.697 & 0.107 \\
\hline WBC & 0.296 & 0.589 \\
\hline Na & 0.618 & 0.435 \\
\hline NVI $(n=121)$ & & \\
\hline TB & 13.544 & 0.000 \\
\hline PTA & 4.417 & 0.039 \\
\hline INR & 7.051 & 0.009 \\
\hline Cr & 6.633 & 0.012 \\
\hline WBC & 1.250 & 0.267 \\
\hline Na & 6.098 & 0.016 \\
\hline
\end{tabular}

4. Comparison of prognostic models between the HVI and NVI groups

Five prognostic model scores were used to assess the 28-day, 90-day, and 1-year mortality prediction of ACLF patients in the two groups. In the NVI group, the MELD model had the highest receiver operating characteristic (ROC) curve (28-day: 0.730, 90-day: 0.722, 1-year: 0.714), and the ROC in other four scores were lower than 0.7. Furthermore, for patients in the HVI group, lactate-free AARC had the highest ROC (90-day: 0.741, 1-year: 0.758), followed by MELD (28-day: 0.723, 90-day: 0.719, 1-year: 0.726), and CLIFACLF (90-day: 0.712, 1-year: 0.700) (Table 5, Figs. 5 and 6). 
Table 5

Comparison of prediction of five scoring models on 28-day, 90-day, and 1-year mortality in two groups

28-day mortality

Variables 90-day mortality

1-year mortality

$N V I(n=121)$

$\begin{array}{lllllll}\text { CTP } & 0.681 & 0.015 & 0.622 & 0.041 & 0.618 & 0.040 \\ & (0.539- & & (0.504- & & (0.505- & \\ & 0.823) & & 0.740) & & 0.731) & \\ \text { CILF-SOFA } & 0.585 & 0.253 & 0.599 & 0.097 & 0.603 & 0.074 \\ & (0.448- & & (0.487- & & (0.494- & \\ & 0.721) & & 0.711) & & 0.712) & \\ \text { CLIF-ACLF } & 0.644 & 0.052 & 0.573 & 0.222 & 0.579 & 0.172 \\ & (0.509- & & (0.457- & & (0.469- & \\ & 0.779) & & 0.689) & & 0.688) & \\ \text { Lactate free } & 0.607 & 0.149 & 0.641 & 0.018 & 0.610 & 0.056 \\ \text { AARC } & (0.463- & & (0.528- & & (0.500- & \\ & 0.751) & & 0.753) & & 0.720) & \\ & 0.730 & 0.002 & 0.722 & 0.000 & 0.714 & 0.000 \\ \text { MELD } & 0.600- & & (0.619- & & (0.611- & \\ & 0.860) & & 0.826) & & 0.817) & \end{array}$

$\mathrm{HVI}(\mathrm{n}=82)$

CTP

0.603

0.175

0.571

0.279

0.617

0.071

(0.460-

0.746 )

(0.443-

0.699)

(0.493-

$0.741)$

CILF-SOFA

0.622

0.110

0.637

$0.036 \quad 0.640$

0.030
(0.488-
$0.755)$
(0.516-
0.758 )
(0.521-
$0.760)$

$\begin{array}{lllllll}\text { CLIF-ACLF } & 0.638 & 0.070 & 0.712 & 0.001 & 0.700 & 0.002 \\ & \begin{array}{llll}0.504- \\ 0.772)\end{array} & & (0.601- & & (0.586- & \\ & & 0.823) & & 0.813) & \\ \text { Lactate free } & 0.714 & 0.005 & 0.741 & 0.000 & 0.758 & 0.000 \\ \text { AARC } & (0.588- & & (0.635- & & (0.655- & \\ & 0.740) & & 0.848) & & 0.861) & \end{array}$




\begin{tabular}{|c|c|c|c|c|c|c|}
\hline \multirow[b]{2}{*}{ MELD } & \multicolumn{2}{|c|}{ 28-day mortality } & \multicolumn{2}{|c|}{ 90-day mortality } & \multicolumn{2}{|c|}{ 1-year mortality } \\
\hline & 0.723 & 0.003 & 0.719 & 0.001 & 0.726 & 0.000 \\
\hline & $\begin{array}{l}(0.602- \\
0.843)\end{array}$ & & $\begin{array}{l}(0.603- \\
0.834)\end{array}$ & & $\begin{array}{l}(0.6154 \\
0.839)\end{array}$ & \\
\hline
\end{tabular}

\section{Discussion}

ACLF is a clinical syndrome characterized by acute deterioration of liver function based on chronic liver disease which is associated with poor outcome in response to an acute insult[1]. At present, there is no consistent definition of ACLF owing to the different etiology of chronic liver disease between the Asian and European populations[21]. Chronic hepatitis B is the main cause of chronic liver disease in Asia, especially in China, while alcoholic liver disease is the most common cause in Europe and USA[22, 23]. In addition, the prognosis of ACLF with various PEs and different underlying chronic in this study were enrolled according to the APASL criteria of ACLF, from three medical centers of China. Among all PEs, HBV reactivation was the leading one (39.9\%), followed by infection (29.6\%). For patients with HBV-ACLF, acute liver deterioration induced by hepatitis $B$ virus exacerbation was still the predominant factor[24] and the mechanism of liver injury was different from other factors, which should be analyzed separately.

There have been previous studies on the PEs of ACLF, in which PEs were divided into hepatic-ACLF and extrahepatic-ACLF groups[4]. The former was induced by hepatic insults such as acute exacerbation or flare-up of $\mathrm{CHB}$, superimposed infection of HAV or HEV, active drinking of alcohol, and use of hepatotoxic drugs; whereas, the extra hepatotropic insults were considered as bacterial infection, UGIB, and other surgical factors. However, it was difficult to simply divide the PEs into hepatic and extrahepatic insults, because the extent of alcohol consumption and duration of active drinking to be included as acute insult was unclear. With prolonged drinking time, autoimmune injury will gradually manifest besides direct liver injury[8, 25]. The proportion of drug insult was $10.3 \%$ in our study, which was consistent with a previous report of the AARC database[8]. Although drugs have been listed as a precipitating factor in ACLF, different types of drugs have heterogeneous effects on liver injury. In this study, bacterial infection as a $\mathrm{PE}$ also accounted for a higher proportion, whereas various bacterial and fungal infections may directly affect the liver in addition to spreading from extrahepatic organs[26]. It remains to be discussed whether hemorrhage or manifestation of liver failure is the PE[27, 28]. In our study, both cases of hemorrhagic events occurred before liver function damage, which may be related to acute hepatic ischemia and subsequent bacterial infection. Overwork was included as a precipitating event in this study, which was not mentioned in previous studies. $7.9 \%$ of all patients had a definite history of overwork in the last month before admission, as shown by a retrospective investigation, wherein overwork was manifested as physical work in excess of normal time and intensity, mental stress, and sleep disorders. In previous studies, overwork is a serious public health problem and links to cardiovascular and cerebrovascular illnesses and deaths[29,30]. Overwork may also be a cause for disease progression by mental stress and fatigue in CHB patients, and the likely potential for liver injury may be related to psychological stress and 
changes in the internal environment[31]. The effects of these heterogeneous factors on liver deterioration in patients with underling hepatitis B or cirrhosis were varied, but they were distinguished from hepatitis B virus reactivation. Therefore, we classified them as NVIs.

ACLF cases in the HVI group were related to recurrence and flare-up of HBV after withdrawal of antiviral drugs, immunosuppressive drugs, and viral mutations. There were no significant intergroup differences in the proportion of ACLF patients with respect to cirrhosis and non-cirrhosis. However, exacerbation time in the HVI group was significantly longer than in the NVI group. It took longer for liver deterioration induced by reactivation of HBV in the HVI group than in that NVI group. At the time of ACLF diagnosis, there was no significant difference in parameters of liver function between the two groups except that serum albumin level was lower in the NVI than HVI group. Jaundice and coagulopathy, which define liver failure, were the main manifestations of organ failure, and the proportion of extrahepatic organ failure was similar in the two groups.

The 90-day and 1-year survival in the HVI group was lower than that in NVI group; there was no difference in the 28-day mortality. The results of this study are inconsistent with those published by Shi et al., which may likely be due to the different ACLF diagnostic criteria and study population. Our study only focused on the HBV-ACLF patients with the ACLF criteria of APASL. The 90-day and long prognosis of liver failure induced by HVI were worse in this study. For HBV-ACLF, effective control of HBV replication makes sense for the recovery of liver injury induced by HBV reactivation[32]. Early and rapid reduction of HBV titer is the essence of therapy, which is related to the suppression of hepatocyte inflammatory necrosis[33]. However, it is suggested that for HBV-ACLF patients, the basic liver function at the start of antiviral treatment is more important for prognosis than the antiviral regimens. Some studies suggested that antiviral therapy was beneficial to ACLF patients with MELD scores of 20-30[34]. Therefore, baseline liver status may have a positive impact on the prognosis of ACLF patients with HBV reactivation. In this study, cirrhosis, bilirubin, PTA, age, and serum creatinine at baseline were independent predictors for the 28- and 90-day mortality.

Patients with non-virus precipitants such as bacterial infection, alcohol consumption, hepatotoxic drugs, and bleeding could benefit from elimination of these causative factors, by considering antibiotic therapy[6], quitting drinking, taking drugs for interruption of liver damage, or undergoing hemostasis therapy. Therefore, the short-term therapy may have an impact on the survival and death of patients. A previous study reported that the patient's condition during the first 3-7 days after ACLF diagnosis determined their prognosis[35]; therefore, we focused on the parameter changes within one week after diagnosis due to their positive predictive prognosis. In this study, there were significant dynamic changes in bilirubin, PTA, INR, creatinine, and blood sodium within 14 days between the patients who survived and those that died in the non-HBV insult group, but there was no significance seen in the HBV insult group. We speculated that the dynamic changes of liver and renal function in the NVI group within 14 days after admission might be related to the prognosis. 
We attempted to compare the predictive scoring model for HBV-ACLF patients induced by hepatotropic virus insult. The MELD score, which is used to assess end-stage liver disease, had good predictive value both for short- and long-term mortality in the two groups. The results revealed that MELD score was superior to the other five models in predicting the prognosis of patients in the NVI group. Lactate-free AARC score showed higher predictive value than for the existing models for the 28-day, 90-day, and 1-year mortality in the NVI group. The AARC score, which included TBil, INR, grade of HE, plasma lactate, and serum creatinine, could reliably predict the severity and outcome for ACLF patients. However, serum lactate test is not a routine test in some countries. A study on the predictive value of lactate-free AARC score among 749 patients with alcoholic liver disease and liver failure (from the AARC database)[36] suggested that the AUC prediction values for 90 -day mortality is similar to that of other models. The lactate-free AARC score could also reflect liver failure to some extent, which is at the core of ACLF, based on APASL criteria. In the HVI group, we found that the lactate-free AARC score had an ideal AUROC and could better predict the 28-day, 90-day, and 1-year mortality than MELD and CLIF-ACLF.

There are some limitations in this study. First, although patients were enrolled from three Chinese hospitals, the sample size was still relatively small. In this study, part of the screened HBV-ACLF patients lacked the $\mathrm{PE}$, and some patients had ambiguous insults; both these categories of patients were excluded. Consequently, there are some deviations in the results of this study. Second, owing to the lack of lactate data, we scored both groups using lactate-free AARC. Therefore, it is necessary to expand the sample size to validate the results reported.

In summary, HBV reactivation is still the most common PE in HBV-ACLF patients, and the 90-day and 1year prognosis of patients with HBV-reactivation-induced HBV-ACLF was worse than that induced by non-virus insult. It is necessary to pay attention to the liver damage caused by HBV reactivation during the management of patients with chronic HBV infection. Lactate-free AARC score and MELD scores are valuable predictors of short- and long-term prognosis in patients with HVI-induced HBV-ACLF.

\section{Declarations}

\section{Ethics approval and consent to participate}

This study was approved by the Ethics Committee of Tianjin Third Central Hospital and informed consent was obtained from all patients prior to study initiation.

\section{Consent for publication}

Not applicable

\section{Availability of data and materials}

All data generated or analyzed during this study are included in this published article.

\section{Competing interests}


The authors declare that they have no competing interests

\section{Funding}

The study was supported by the National 13th 5-year Plan for Hepatitis Research (2017ZX10203201-007) and the National Natural Science Foundation of China (No.81870429凶

\section{Authors's contributions}

$\mathrm{JL}$ and TH designed the research. JL and $\mathrm{LL}$ drafted the manuscript. QZ and HL collected the data and established the database. YYC and FL presided over the enrollment and exclusion of the research subjects. JL and JL analyzed the data statistically. YC, ZPD, MMX, SJX, SLY and FJS managed and followed up the patients. TH participated in paper modification and revised the manuscript for English writing. All authors read and approved the final manuscript.

\section{Acknowledgements}

Not applicable

\section{References}

1. Wu T, Li J, Shao L, Xin J, Jiang L, Zhou Q, Shi D, Jiang J, Sun S, Jin L et al: Development of diagnostic criteria and a prognostic score for hepatitis B virus-related acute-on-chronic liver failure. Gut 2018, 67(12):2181-2191.

2. Moreau R, Jalan R, Gines P, Pavesi M, Angeli P, Cordoba J, Durand F, Gustot T, Saliba F, Domenicali M et al: Acute-on-chronic liver failure is a distinct syndrome that develops in patients with acute decompensation of cirrhosis. Gastroenterology 2013, 144(7):1426-1437, 1437 e1421-1429.

3. Zhang Q, Li Y, Han T, Nie C, Cai J, Liu H, Liu Y: Comparison of current diagnostic criteria for acute-onchronic liver failure. PloS one 2015, 10(3):e0122158.

4. Shi Y, Yang Y, Hu Y, Wu W, Yang Q, Zheng M, Zhang S, Xu Z, Wu Y, Yan H et al: Acute-on-chronic liver failure precipitated by hepatic injury is distinct from that precipitated by extrahepatic insults. Hepatology 2015, 62(1):232-242.

5. Yin S, Wang SJ, Gu WY, Zhang Y, Chen LY, Li H: Risk of different precipitating events for progressing to acute-on-chronic liver failure in HBV-related cirrhotic patients. Journal of digestive diseases 2017, 18(5):292-301.

6. Fernandez J, Acevedo J, Wiest R, Gustot T, Amoros A, Deulofeu C, Reverter E, Martinez J, Saliba F, Jalan $\mathrm{R}$ et al: Bacterial and fungal infections in acute-on-chronic liver failure: prevalence, characteristics and impact on prognosis. Gut 2018, 67(10):1870-1880.

7. Li H, Chen LY, Zhang NN, Li ST, Zeng B, Pavesi M, Amoros A, Mookerjee RP, Xia Q, Xue F et al: Characteristics, Diagnosis and Prognosis of Acute-on-Chronic Liver Failure in Cirrhosis Associated to Hepatitis B. Scientific reports 2016, 6:25487. 
8. Sarin SK, Choudhury A, Sharma MK, Maiwall R, Al Mahtab M, Rahman S, Saigal S, Saraf N, Soin AS, Devarbhavi $\mathrm{H}$ et al: Acute-on-chronic liver failure: consensus recommendations of the Asian Pacific association for the study of the liver (APASL): an update. Hepatology international 2019, 13(4):353390.

9. [The guidelines of prevention and treatment for chronic hepatitis B (2019 version)]. Zhonghua gan zang bing za zhi $=$ Zhonghua ganzangbing zazhi $=$ Chinese journal of hepatology 2019, 27(12):938961.

10. Weissenborn K: Hepatic Encephalopathy: Definition, Clinical Grading and Diagnostic Principles. Drugs 2019, 79(Suppl 1):5-9.

11. Loomba R, Liang TJ: Hepatitis B Reactivation Associated With Immune Suppressive and Biological Modifier Therapies: Current Concepts, Management Strategies, and Future Directions. Gastroenterology 2017, 152(6):1297-1309.

12. Gawande A, Gupta GK, Gupta A, Wanjari SJ, Goel V, Rathore V, Bhardwaj H, Nijhawan S: Acute-onChronic Liver Failure: Etiology of Chronic and Acute Precipitating Factors and Their Effect on Mortality. Journal of clinical and experimental hepatology 2019, 9(6):699-703.

13. Devarbhavi H, Choudhury AK, Sharma MK, Maiwall R, Al Mahtab M, Rahman S, Chawla YK, Dhiman RK, Duseja A, Taneja S et al: Drug-Induced Acute-on-Chronic Liver Failure in Asian Patients. The American journal of gastroenterology 2019, 114(6):929-937.

14. Bajaj JS, O'Leary JG, Reddy KR, Wong F, Olson JC, Subramanian RM, Brown G, Noble NA, Thacker LR, Kamath PS: Second infections independently increase mortality in hospitalized patients with cirrhosis: the North American consortium for the study of end-stage liver disease (NACSELD) experience. Hepatology 2012, 56(6):2328-2335.

15. Virtanen M, Jokela M, Madsen IE, Magnusson Hanson LL, Lallukka T, Nyberg ST, Alfredsson L, Batty GD, Bjorner JB, Borritz $M$ et al: Long working hours and depressive symptoms: systematic review and meta-analysis of published studies and unpublished individual participant data. Scandinavian journal of work, environment \& health 2018, 44(3):239-250.

16. Lin RT, Lin CK, Christiani DC, Kawachi I, Cheng Y, Verguet S, Jong S: The impact of the introduction of new recognition criteria for overwork-related cardiovascular and cerebrovascular diseases: a crosscountry comparison. Scientific reports 2017, 7(1):167.

17. Lebosse F, Guillaud O, Forestier J, Ecochard M, Boillot O, Roman S, Mion F, Dumortier J: Prognostic Value of Metabolic Liver Function Tests: a Study on 711 Cirrhotic Patients. Journal of gastrointestinal and liver diseases: JGLD 2016, 25(3):337-343.

18. Freeman RB, Jr.: Model for end-stage liver disease (MELD) for liver allocation: a 5-year score card. Hepatology 2008, 47(3):1052-1057.

19. Jalan R, Saliba F, Pavesi M, Amoros A, Moreau R, Gines P, Levesque E, Durand F, Angeli P, Caraceni P et al: Development and validation of a prognostic score to predict mortality in patients with acute-onchronic liver failure. Journal of hepatology 2014, 61(5):1038-1047. 
20. Choudhury A, Jindal A, Maiwall R, Sharma MK, Sharma BC, Pamecha V, Mahtab M, Rahman S, Chawla YK, Taneja $S$ et al: Liver failure determines the outcome in patients of acute-on-chronic liver failure (ACLF): comparison of APASL ACLF research consortium (AARC) and CLIF-SOFA models. Hepatology international 2017, 11(5):461-471.

21. Sarin SK, Choudhury A: Management of acute-on-chronic liver failure: an algorithmic approach. Hepatology international 2018, 12(5):402-416.

22. Zhao RH, Shi Y, Zhao H, Wu W, Sheng JF: Acute-on-chronic liver failure in chronic hepatitis $B$ : an update. Expert review of gastroenterology \& hepatology 2018, 12(4):341-350.

23. Gustot $T$, Jalan R: Acute-on-chronic liver failure in patients with alcohol-related liver disease. Journal of hepatology 2019, 70(2):319-327.

24. Lei JH, Peng F, Chen Z, Xiao XQ: Is HBV viral load at admission associated with development of acute-on-chronic liver failure in patients with acute decompensation of chronic hepatitis $B$ related cirrhosis? BMC infectious diseases 2019, 19(1):363.

25. Wasmuth HE, Kunz D, Yagmur E, Timmer-Stranghoner A, Vidacek D, Siewert E, Bach J, Geier A, Purucker EA, Gressner AM et al: Patients with acute on chronic liver failure display "sepsis-like" immune paralysis. Journal of hepatology 2005, 42(2):195-201.

26. Strnad P, Tacke F, Koch A, Trautwein C: Liver - guardian, modifier and target of sepsis. Nature reviews Gastroenterology \& hepatology 2017, 14(1):55-66.

27. Amitrano L, Guardascione MA, Martino R, Manguso F, Menchise A, Balzano A: Hypoxic hepatitis occurring in cirrhosis after variceal bleeding: still a lethal disease. Journal of clinical gastroenterology 2012, 46(7):608-612.

28. Bruns T, Zimmermann HW, Stallmach A: Risk factors and outcome of bacterial infections in cirrhosis. World journal of gastroenterology 2014, 20(10):2542-2554.

29. Yamauchi T, Yoshikawa T, Sasaki T, Matsumoto S, Takahashi M, Suka M, Yanagisawa H: Cerebrovascular/cardiovascular diseases and mental disorders due to overwork and work-related stress among local public employees in Japan. Industrial health 2018, 56(1):85-91.

30. Kim I, Koo MJ, Lee HE, Won YL, Song J: Overwork-related disorders and recent improvement of national policy in South Korea. Journal of occupational health 2019, 61(4):288-296.

31. Jang Y, Boo S, Yoo H: Hepatitis B Virus Infection: Fatigue-Associated Illness Experiences Among Koreans. Gastroenterology nursing: the official journal of the Society of Gastroenterology Nurses and Associates 2018, 41(5):388-395.

32. Wang J, Ma K, Han M, Guo W, Huang J, Yang D, Zhao X, Song J, Tian D, Qi J et al: Nucleoside analogs prevent disease progression in HBV-related acute-on-chronic liver failure: validation of the TPPM model. Hepatology international 2014, 8(1):64-71.

33. Ma K, Guo W, Han M, Chen G, Chen T, Wu Z, Yang D, Huang J, Huang Y, Zhao X et al: Entecavir treatment prevents disease progression in hepatitis $B$ virus-related acute-on-chronic liver failure: establishment of a novel logistical regression model. Hepatology international 2012, 6(4):735-743. 
34. Yuen MF: Anti-viral therapy in hepatitis B virus reactivation with acute-on-chronic liver failure. Hepatology international 2015, 9(3):373-377.

35. Gustot T, Fernandez J, Garcia E, Morando F, Caraceni P, Alessandria C, Laleman W, Trebicka J, Elkrief L, Hopf $C$ et al: Clinical Course of acute-on-chronic liver failure syndrome and effects on prognosis. Hepatology 2015, 62(1):243-252.

36. Dong Joon Kim TYK AKC, Ji Dong Jia, Shiv K Sarin, Priyanka Jain,et al.: Usefulness of lactate-free Asian Pacific Association for the study of Liver acute-on-chronic liver failure (AARC)-ACLF score for prediciting short-term mortality in patients with alcoholic liver diseas. 27th Annual Conference Asian Pacific Association for the Study of liver 2018, PO-C-06.

\section{Figures}

452 Patients hospitalized for ACLF were screened

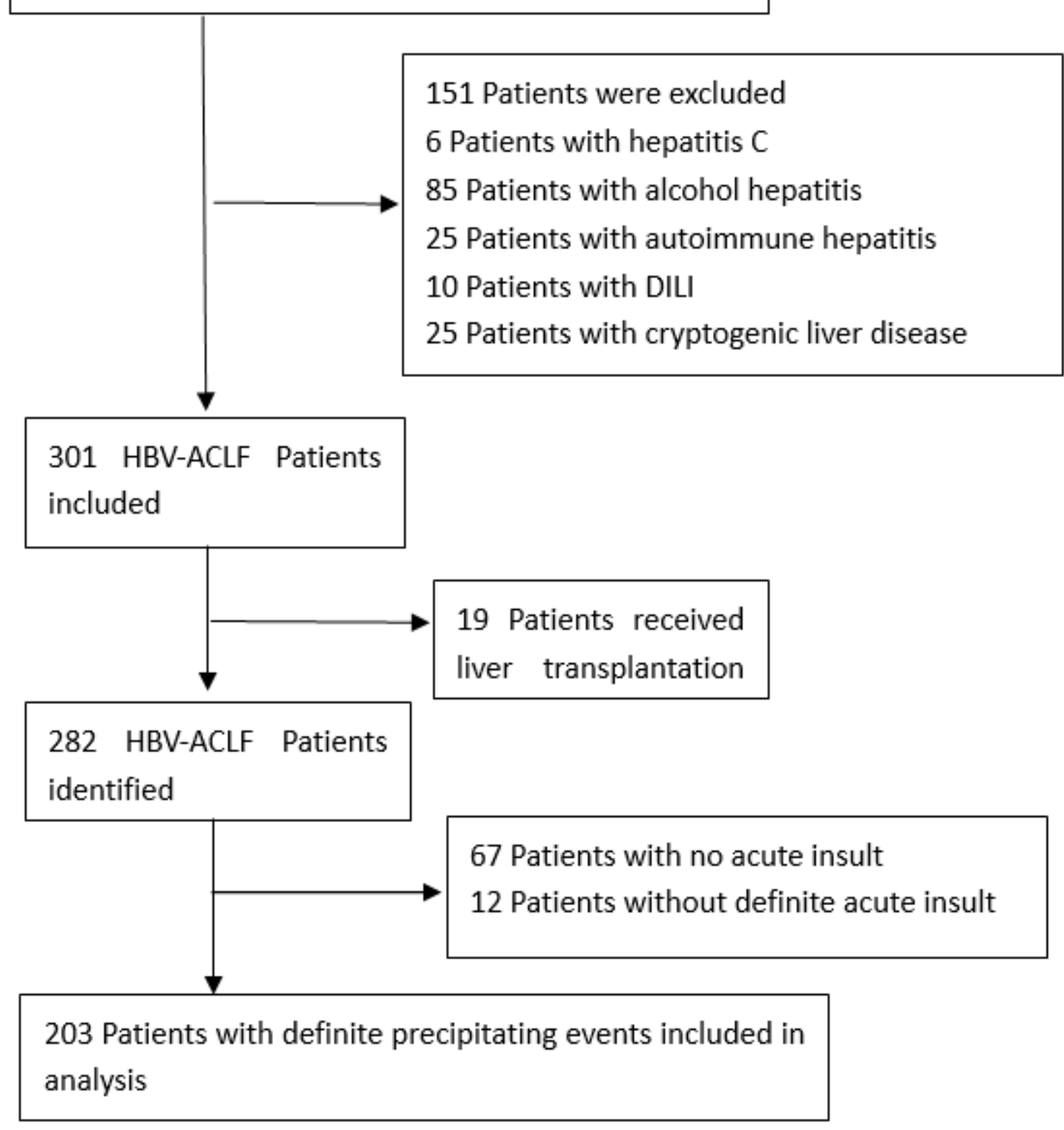


Figure 1

Flowchart of enrollment of HBV-related ACLF cohort
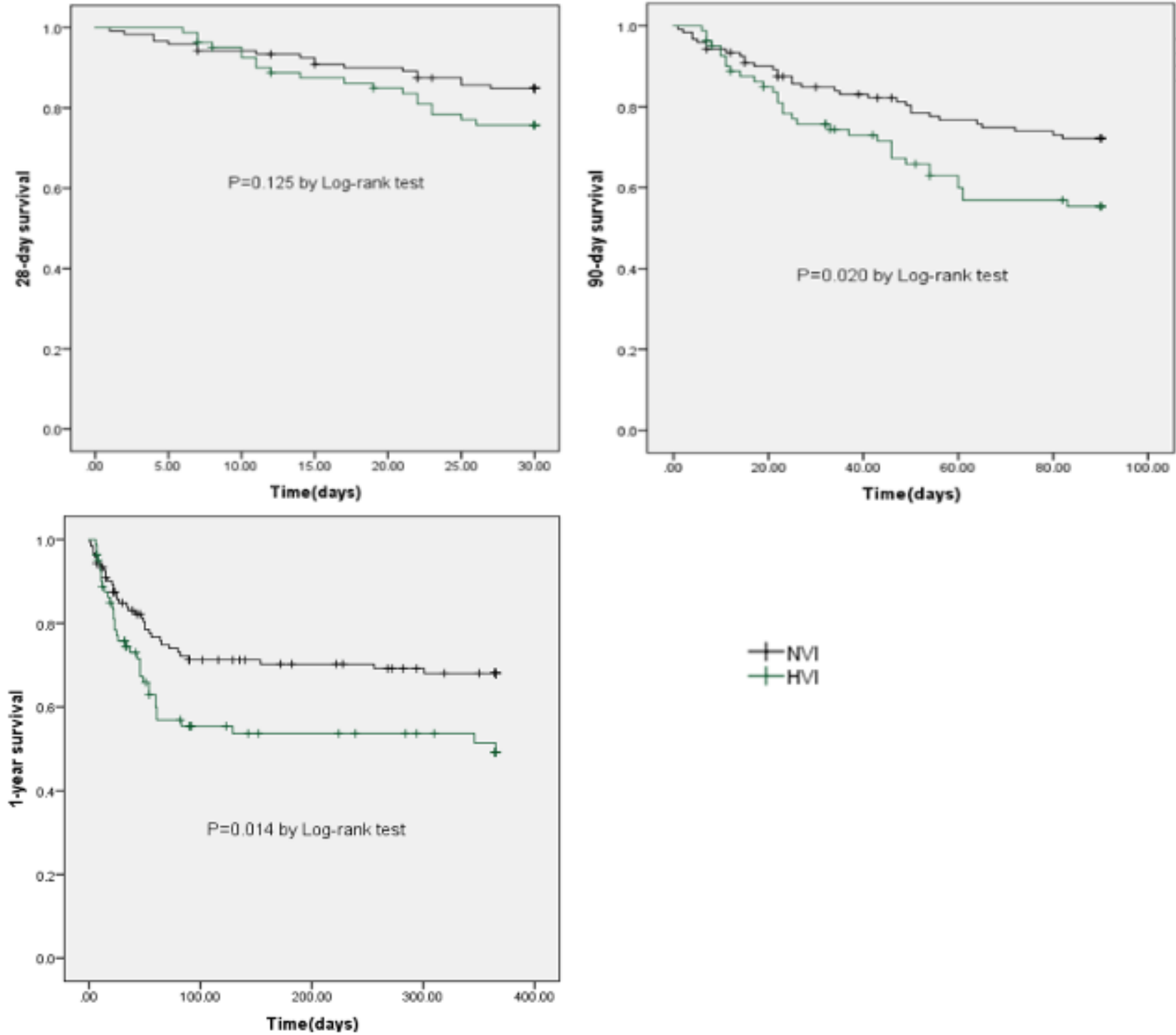

\section{Figure 2}

Comparison of the 28-day, 90-day and 1-year survival between hepatotropic viral insult group and nonvirus insult group 

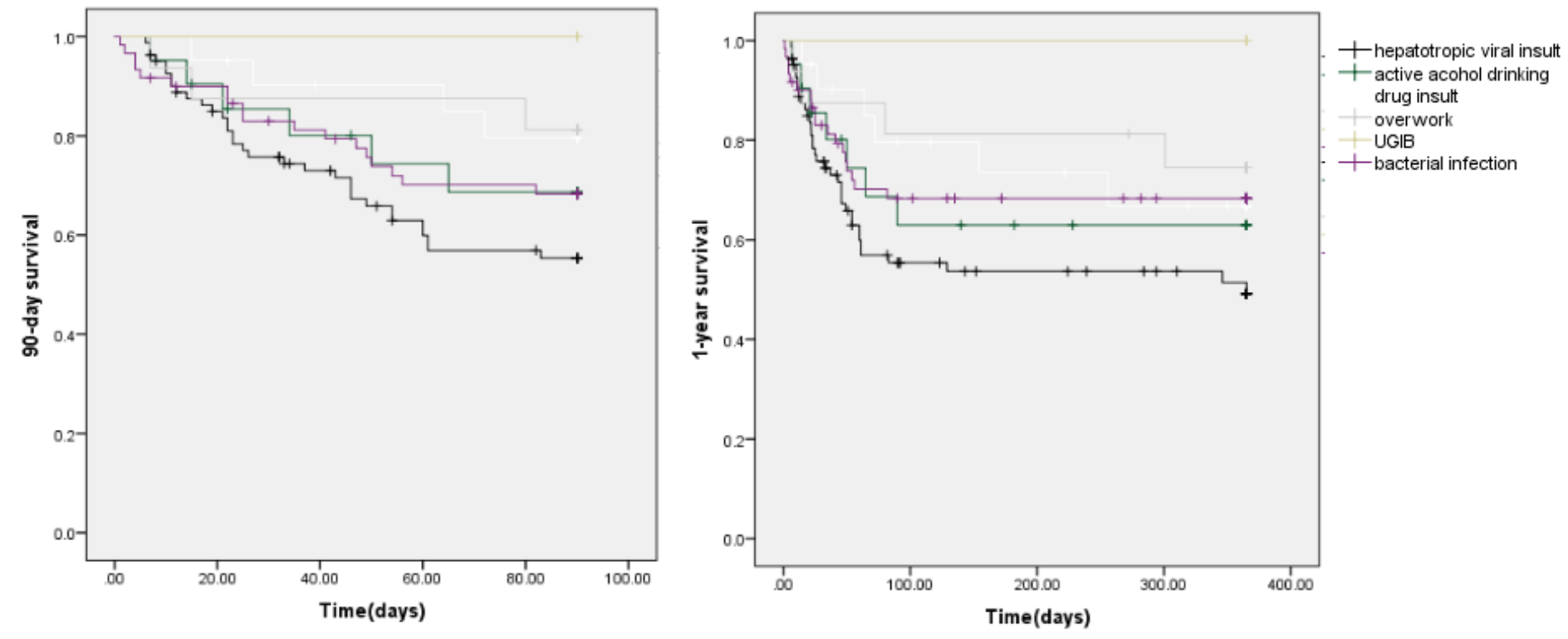

Figure 3

Comparison of the 90 day and 1-year survival of patients induced by hepatotropic viral insult and active alcohol drinking, hepatotoxic drugs, overwork, UGIB and bacterial infection group
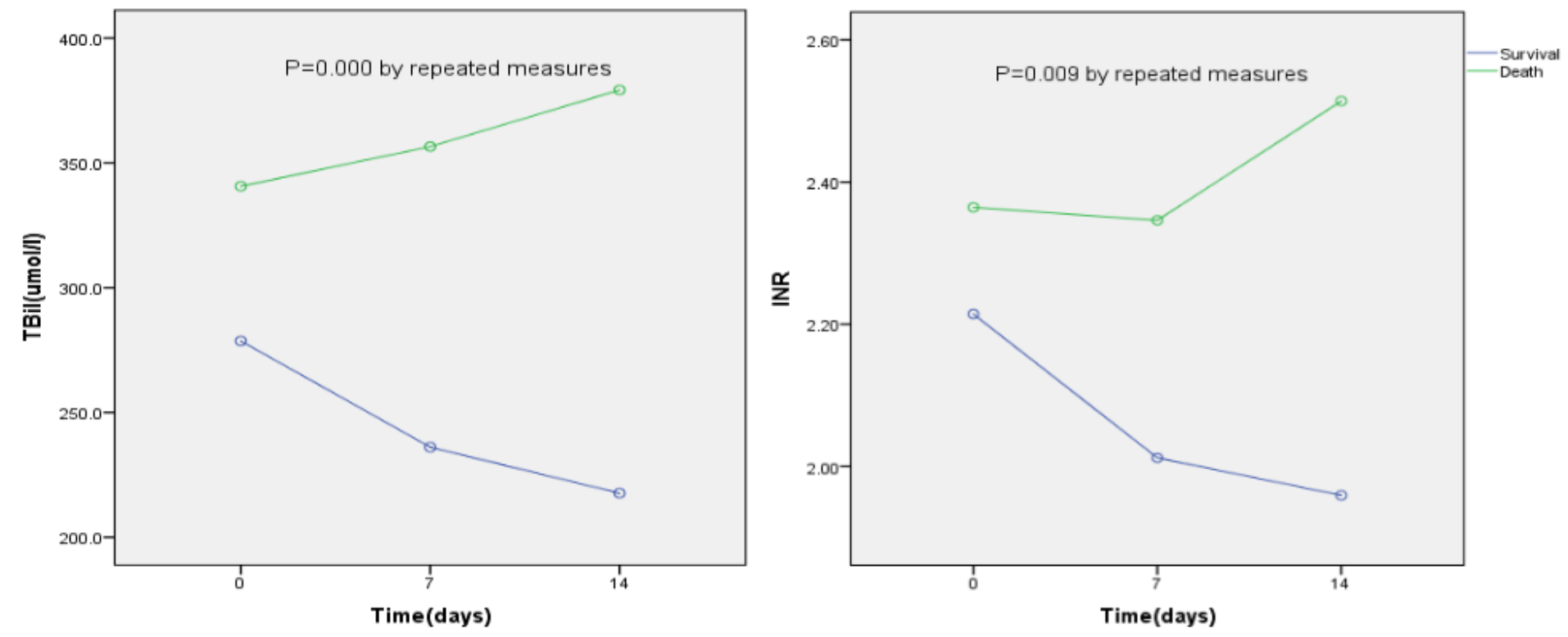

Figure 4

Dynamic changes of TBil and INR within 14 days after ACLF diagnosis of NVI group 4. Comparison of prognostic models between the $\mathrm{HVI}$ and $\mathrm{NVI}$ groups 

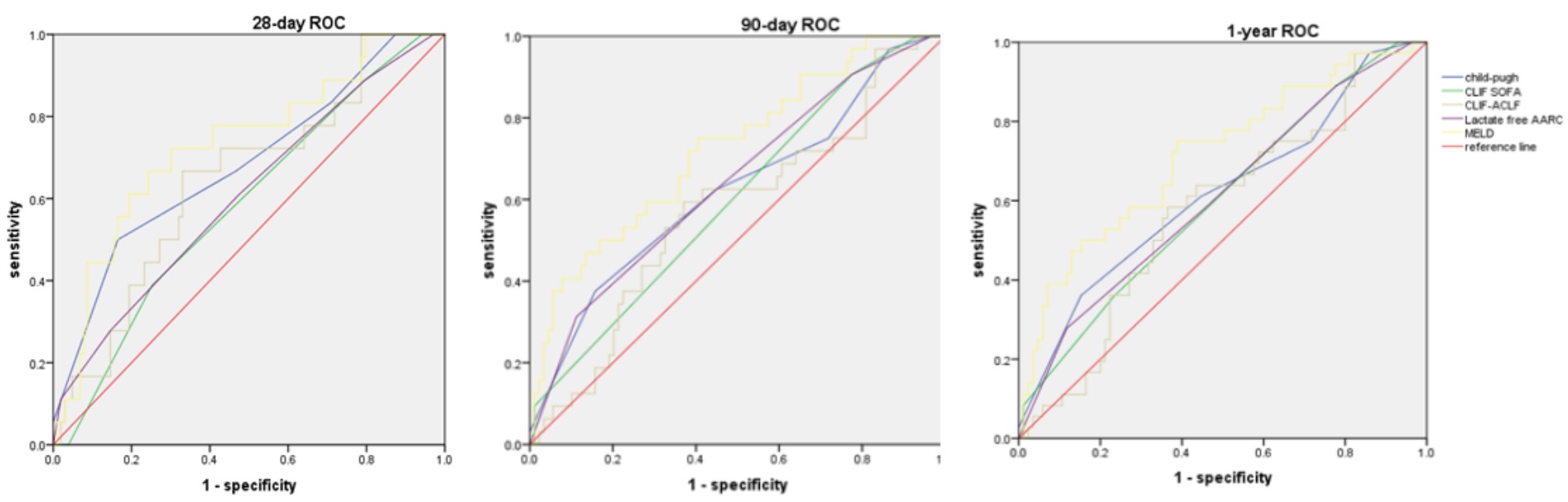

\section{Figure 5}

ROC curve of prognostic models in 28-day, 90-day and 1-year mortality in NVI group
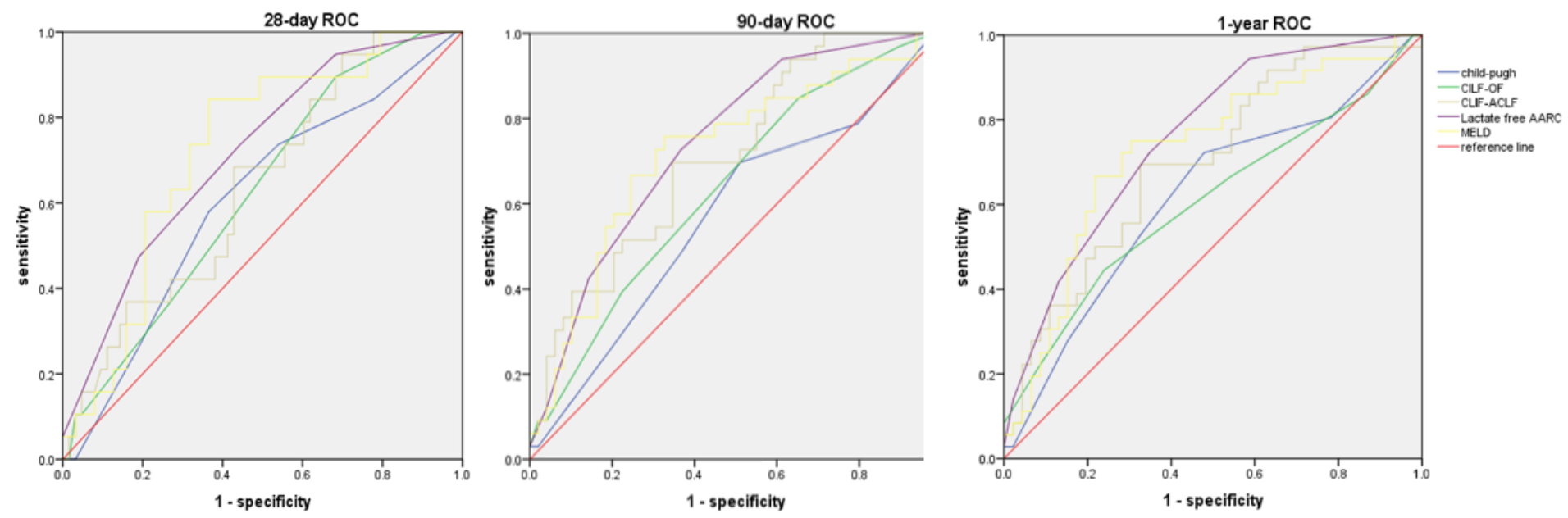

Figure 6

ROC curve of prognostic models in 28-day, 90-day and 1-year mortality in HVI group 